\title{
Annexin A2 is implicated in multi-drug-resistance in gastric cancer through p38MAPK and AKT pathway
}

\author{
Z. D. ZHANG, Y. LI*, L. Q. FAN, Q. ZHAO, B. B. TAN, X. F. ZHAO \\ Department of General Surgery, the Fourth Affiliated Hospital, Hebei Medical University, 12 Jian-Kang St., 050011 Shijiazhuang, China \\ ${ }^{*}$ Correspondence: li_yong_hbth@126.com
}

Received November 30, 2013 / Accepted March 12, 2014

\begin{abstract}
Studies have shown that Annexin A2 (ANXA2) is related with tumor proliferation, apoptosis, differentiation, invasion, migration, and drug resistance. The purpose of this study was to investigate the role and its mechanisms of ANXA2 in multidrug-resistance (MDR) in gastric cancer. ANXA2 expression in both gastric cancer tissues and cell lines were detected by quantitative real-time PCR (RT-qPCR) and Western blotting. The cell proliferation was measured by SRB assay. The pool of siRNA against ANXA2 was designed and synthesized and then transfected into resistant gastric cancer SGC7901/DDP cells. ANXA2 expression was detected by RT-qPCR and Western blotting. Drug sensitivities of SGC7901/DDP cells to P-gp-related drug (doxorubicin) and P-gp-non-related drugs (5-FU and cisplatin) were measured by SRB assay.

Expression of MDR-related genes and phosphorylation of AKT and MAPKs were also detected by RT-qPCR and Western blotting. Results showed that ANXA2 expression was significantly higher in gastric specimens than that in normal tissues, and negatively correlated with the differentiation level of gastric cancer. In addition, ANXA2 expression level was higher in SGC7901/DDP cells than that in parent SGC7901 cells. After knock-down ANXA2 expression using ANXA2 small interfering RNA, the drug sensitivity of SGC7901/DDP cells to doxorubicin, 5-FU and DDP increased. Delivery of ANXA2 siRNA significantly downregulated the expression of P-gp, MRP1 and Bcl-2, while markedly upregulated Bax in SGC7901/DDP cells. However, several other MDR factors such as GST- $\pi$, TOPO-I and TOPO-II had no obvious changes. Additionally, phosphorylation of P38MAPK and AKT, but not ERK1/2 or JNKs was specifically decreased in SGC7901/DDP cells after ANXA2 siRNA delivery. Importantly, P38MAPK and AKT inhibitor increased the drug sensitivity of SGC701/DDP cells in a similar way as ANXA2 siRNAs does. ANXA2 is involved in gastric cancer MDR through regulating p38MAPK and AKT pathways as well as certain MDR factors.
\end{abstract}

Key words: gastric cancer, Annexin A2, siRNA, multidrug resistance, SGC7901/DDP cells

Globally, gastric cancer(GC) is the second mostly frequent cause of cancer-related mortality and the incidence is much higher in Asia than other geographic areas [1,2]. In China, GC is the second leading cause of cancer-related mortality and has the third highest incidence [3]. Because GC is usually diagnosed in the advanced or metastatic stage, the prognosis of gastric cancer is generally rather poor, with a 5 -year survival rate of less than $30 \%[1,4]$. Surgical treatment remains the standard therapy strategy at present, but recurrence appears in approximately $50-70 \%$ of patients with advanced disease [5]. Therefore, chemotherapy has been widely accepted as palliative treatment for both resectable and advanced GC, leading to improvements in quality of life for patients and prolonged survival [6]. Although GC is considered as relatively insensitive to chemotherapeutics, several important chemotherapeutic agents such as cisplatin (DDP) and 5-fluorouracil (5-FU) are effective. To date, the survival time is still unsatisfactory so far [7]. Multiple lines of evidence suggest that long-term chemotherapy often fails to eliminate all cancer cells due to the development of multidrug resistance (MDR), which can further caused the cancer recurrence $[8]$.

Annexin A2 (ANXA2), a calcium dependent phospholipid binding protein, can bind to collagen I, cathepsin B and tenascin-C [9], and is involved in maintaining the plasticity and rearrangement of the actin cytoskeleton [10] . There is overwhelming evidence confirming that ANXA2 is a multifunctional protein which is involved in cell surface fibrinolysis [11], signaling transduction [12], inflammation [13], proliferation [9], apoptosis [14], and tumor progression [15]. 
Increased ANXA2 levels have been observed in malignant tissues of the liver, rectum, colon, breast, ovarian, and stomach [16-20]. Multiple lines of evidence suggest that the expression of ANXA2 is related with differentiation, proliferation, invasion, migration, and apoptosis of cancer cells. More recently, increasing studies of ANXA2 on the resistance of cancer are forthcoming. Therefore, ANXA2 -targeted interference by Small interfering RNA (siRNA) may be an effective therapeutic strategy to mediate the biological behaviors of gastric cancer cells.

In this study, the expression of ANXA2 in gastric cancer and its relationship with cell differentiation were first detected. Then, a DDP resistant gastric cancer cell line SGC7901/DDP was established by using DDP treatment of SGC7901 and the siRNA-targeted ANXA2 were transfected into SGC7901/DDP cells. After transfection, the drug sensitivity of SGC7901/DDP to P-gp-related drug (doxorubicin) and two P-gp-non-related drugs (5-FU and cisplatin) were investigated. In addition, the effects of ANXA2 siRNA transfection on the expression of MDR-related genes and activation of some molecular signaling pathway were also observed.

\section{Patients and methods}

Patients and tissue specimens. All 18 patients with primary GC (I-III stage) were histologically diagnosed in the The Fourth Affiliated Hospital, Hebei Medical University of China in 2012. Tumor stage was defined according to the 7th edition of the AJCC cancer staging manual 2010. None of the patients enrolled in the study had received chemotherapy or radiotherapy before surgery, and there was no evidence of any other malignancies. Patients underwent radical resection, the tumor specimens and distant normal gastric mucous tissues $(>5 \mathrm{~cm}$ from the margin of the tumor) were collected and stored at $-80^{\circ} \mathrm{C}$ for quantitative real-time PCR and Westernblot analysis. Five non-tumor control gastric tissue samples were obtained from patients after radical gastrectomy. The experimental protocols were approved by the Ethics Committee of the The Fourth Affiliated Hospital, Hebei Medical University of China.

Cell culture and treatment. Gastric cancer cell line MKN28, SGC7901 and BGC823, human gastric mucosa epithelial cell line GES- 1 as well as cisplatin (DDP)-resistant cell line SGC7901/DDP were purchased from Cell Resource Center of Life Sciences (Shanghai, China). All cell lines were cultured in RPMI 1640 medium (Gibco, Grand Island, U.S.) supplemented with $10 \%$ FBS, 100 units/ml penicillin and $100 \mathrm{mg} / \mathrm{ml}$ streptomycin. Cells were maintained in a humidified atmosphere of $5 \% \mathrm{CO}_{2}$ at $37^{\circ} \mathrm{C}$ and dissociated with $0.25 \%$ trypsin containing $0.02 \%$ EDTA and then passaged. cisplatin (DDP)-resistant SGC7901/DDP cells were cultured in the medium containing $0.5 \mu \mathrm{g} / \mathrm{ml}$ DDP and grown in DDP-free medium at least 2 weeks before any procedure.

RNA extraction and Real-time quantitative PCR (RTQPCR). Total cellular RNA in the tissue specimens and in cells with different treatments was extracted using the Trizol (Invitrogen) according to the manufacturer's protocol. cDNA was synthesized using random primers and MMLV reverse transcriptase. RT-QPCR were performed according to established protocols on an ABI 7500 PCR system (Applied Biosystems, USA) using the SYBR Green RT-PCR Kit (Applied Biosystems, Foster City, CA). Primer sequences used in PCR are listed in Table 1 . PCR were done by running 40 cycles $\left(94^{\circ} \mathrm{C}\right.$ for $30 \mathrm{~s}$, $60^{\circ} \mathrm{C}$ for $30 \mathrm{~s}$, and $72^{\circ} \mathrm{C}$ for $\left.30 \mathrm{~s}\right)$. Fluorescence was detected at the end of each cycle. The specificity of the products was confirmed by melting curve analysis. The relative expression levels of target genes were carried out using the $2^{-\Delta \Delta \mathrm{Ct}}$ method. All qRT-PCR reactions were conducted in triplicate.

Small interfering RNA (siRNA) treatment. siRNA targeting ANXA 2 and non-targeted siRNA were synthetized by Ribo Bio (Guangzhou, China). The sequences of pool of against ANXA2 and non-targeted control were listed in Table 2; Cells were transfected by using Lipofectamine 2000 (Invitrogen) according to the manufacturer's protocol. The transfected cells were treated with DDP $(0.5 \mu \mathrm{g} / \mathrm{ml})$ for $24 \mathrm{~h}$ to determine the drug resistance.

Sulforhodamine B (SRB) assay. SGC7901 cells and SGC7901/DDP cells were seeded in 96-well culture plates at densities of $1 \times 10^{4}$ cells/well and were allowed to adhere for $24 \mathrm{~h}$ before treatment. Cells were transfected with ANXA2-siRNA or non-targeting siRNA for $24 \mathrm{~h}$, then cellular growth was assessed by SRB assay. after $24 \mathrm{~h}$ incubation period, 10 -fold diluted in five concentrations of DDP, doxorubicin, or 5-FU (original

Table 1. Primer sequences for quantitative PCR.

\begin{tabular}{lll}
\hline Gene & Forward primer $\left(\mathbf{5}^{\prime}\right.$ to $\mathbf{3}^{\prime}$ ) & Reverse primer $\mathbf{( 5}$ ' to $\mathbf{3}^{\prime}$ ) \\
\hline ANXA2 & TAACTTTGATGCTGAGCGGG & TAATTTCCTGCAGCTCCTGG \\
P-gp & GAATGTTCAGTGGCTCCGAG & ACAATCTCTTCCTGTGACACC \\
MRP1 & CATCAGCAGGCACCACAAC & TTCCAGGTCTCCTCCTTCTTG \\
GST- $\pi$ & ATACCATCCTGCGTCACCTG & TCCTTGCCCGCCTCATAGTT \\
TOPO-I & AGGAGGAAGGAGAAACAGCG & TCTGGGTGTAGATTGATGTGCT \\
TOPO-II & GGGTTTACGATGAAGATGTTGG & CTTTGTTTGTTGTCCGCAGC \\
Bcl2 & TGTGTGGAGAGCGTCAACC & TGGATCCAGGTGTGCAGGT \\
Bax & TTTCTGACGGCAACTTCAAC & AGTCCAATGTCCAGCCCAT \\
GAPDH & GACCCCTTCATTGACCTCAAC & CGCTCCTGGAAGATGGTGAT \\
\hline
\end{tabular}


Table 2. siRNA sequences for quantitative PCR.

\begin{tabular}{lll}
\hline Gene & Sense strand (5' to 3') & Antisense strand (5' to 3') \\
\hline Annexin A 2 & GUUACAGCCCUUAUGACAU & AUGUCAUAAGGGCUGUAAC \\
& GAAAGUACGGCAAGUCCCU & AGGGACUUGCCGUACUUUC \\
& GAACUUGCAUCAGCACUGA & UCAGUGCUGAUGCAAGUUC \\
& GAAGAAGGCUUCCUUCAAA & UUUGAAGGAAGCCUUCUUC \\
non-targeted siRNA & UGGUUUACAUGUCGACUAA & \\
& UGGUUUACAUGUUGUGUGA & \\
& UGGUUUACAUGUUUUCUGA & \\
& UGGUUUACAUGUUUUCCUA & \\
\hline
\end{tabular}

concentrations were DDP $0.05 \mu \mathrm{g} / \mathrm{ml}$, doxorubicin 0.001 $\mu \mathrm{g} / \mathrm{ml}$, and 5 -FU $0.025 \mu \mathrm{g} / \mathrm{ml}$, respectively) and were added to the cells. The Lipofectamine 2000 group served as control, and blank group was used. The cells were plated in sextuplicate (6 replicate wells per cell density) and experiments were performed three times. For SRB assay, the culture medium was aspirated and RPMI 1640 medium with 10\% (final concentration) trichloroacetic acid (TCA) and was added to each well for $30 \mathrm{~h}$ at $4^{\circ} \mathrm{C}$ to precipitate the proteins. Then, precipitated proteins were stained for $30 \mathrm{~min}$ at room temperature with $0.4 \%$ $(\mathrm{w} / \mathrm{v})$ SRB in acetic acid solution $1 \% \mathrm{v} / \mathrm{v}$. After washed with deionized water for 5 times, the bound SRB was solubilized in $10 \mathrm{mmol} / \mathrm{l}$ Tris buffer. Finally, absorbance (optical density, OD) was read at a wavelength of $540 \mathrm{~nm}$ on an ELISA plate reader. Half maximal inhibitory concentration $\left(\mathrm{IC}_{50}\right.$ ) was determined by SPSS software version 11.5.

Western blotting analysis. Cells were harvested and lysed on ice in RIPA buffer containing protease inhibitor. Protein concentration was determined using a Bradford method (Bio-Rad, Hercules, USA), and $60 \mu \mathrm{g}$ of protein for each sample was separated by $10 \%$ SDS-PAGE, and transferred to PVDF membranes (Roche). Membranes were incubated overnight at $4^{\circ} \mathrm{C}$ with primary antibodies against ANXA2, P-gp (P-glycoprotein), MRP1 (Multiple Drug Resistant Protein 1), Bcl-2 (B-cell lymphoma 2), Bax, GST- $\pi$ (glutathione-S-transferase $\pi$ ), TOPO-I (Topoisomerase I), TOPO-II (Topoisomerase II), ERK1/2 (Extracellular signal-regulated kinases), phospho-ERK1/2, JNK1/2/3 (c-Jun N-terminal kinase), phospho-JNK1/2/3, p38MAPK (p38 Mitogen-activated protein kinases), phospho-p38MAPK, AKT (RAC-alpha serine/threonine-protein kinase), phospho-AKT, or GAPDH (Glyceraldehyde 3-phosphate dehydrogenase), all primary antibodies were from Santa Cruz, USA. GAPDH was utilized for an endogenous reference to standardize protein expression levels. Bands were detected with chemiluminescence (Pierce). The density of bands was determined by the Gelpro 4.0 software.

Data statistics. Data were represented as Mean \pm Standard Deviation. Statistical Package for the Social Sciences (SPSS) for Windows (version 13.0; SPSS, Chicago, IL, USA) was used for statistical analysis, $\mathrm{p}<0.05$ was considered as statistically significant.

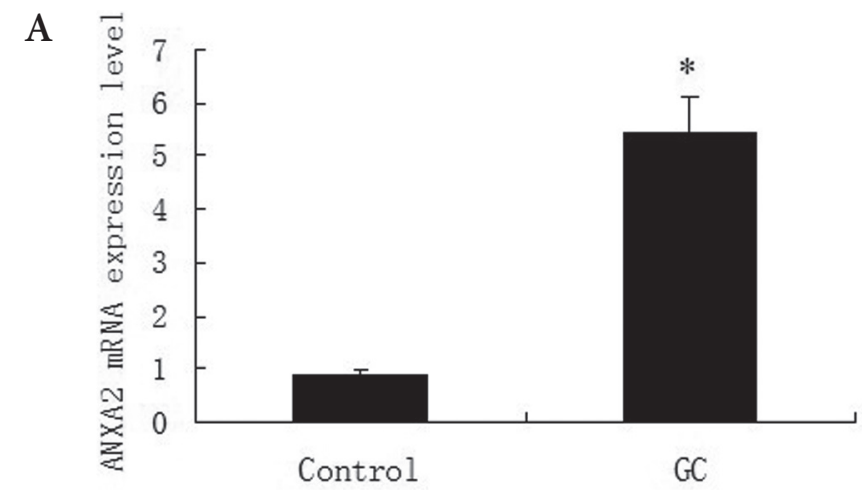

B
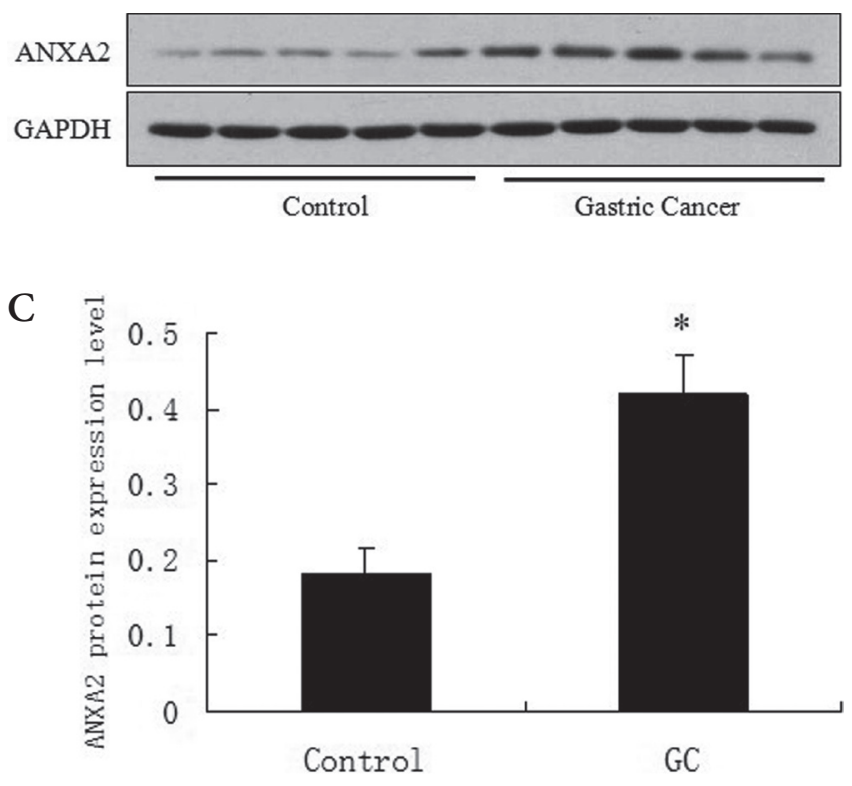

Figure 1. Expression of ANXA2 were up-regulated in gastric cancer tissues. Clinical gastric tumor specimens as well as control normal tissues were obtained and subjected to RT-QPCR (A) and Western blotting (B and C) to determine the expression of ANXA2. For RT-QPCR and Western blotting assays, GAPDH was used for an endogenous reference to standardize the mRNA and protein expression levels. Values are mean \pm S.D. $(\mathrm{n}=3) .{ }^{*}, \mathbf{p}<\mathbf{0 . 0 5}$ compared with cancer-adjacent normal tissues group. The images showed representative images from at least three independent experiments. 


\section{Results}

Annexin A 2 is high-expressed in gastric cancer tissue. Firstly, the normal and gastric specimens were detected for ANXA2 mRNA and protein expression using RT-QPCR and Western-blotting analysis, respectively. All of the assays gave rise to similar results in most cases. ANXA2 mRNA and protein levels were significantly higher in gastric specimens than that in normal tissues ( $p<0.05$, Fig. 1). These results indicated that ANXA2 may play an important role in the development of gastric cancer.

Expression of ANXA2 is negative when correlated with gastric cancer differentiation. To assess whether ANXA2 were related with GC differentiation, the expression of ANXA2 in GC tissues with different stage of differentiation were further detected by RT-QPCR and Western blotting analysis. And the results showed that the higher differentiation stage, the lower expression level of ANXA2. Results were shown in Fig 2 A,
A

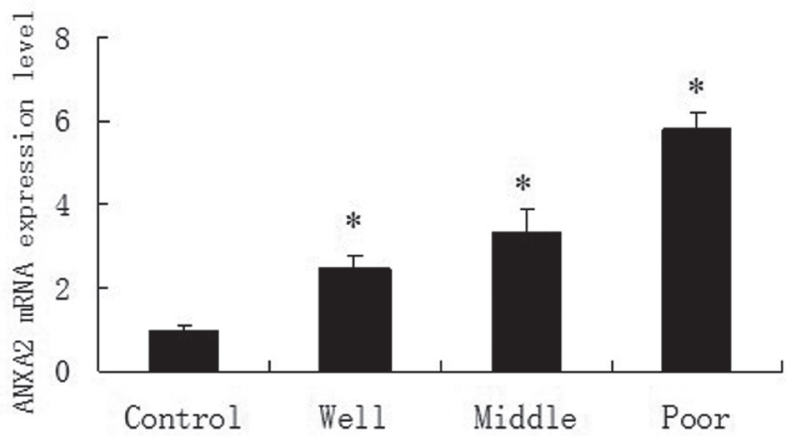

C

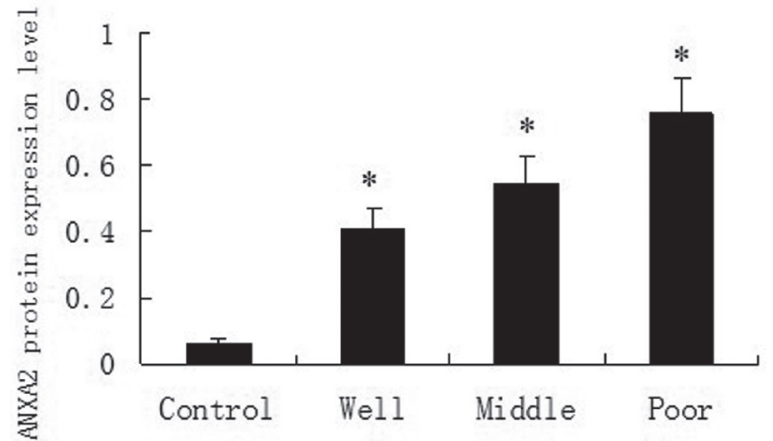

E

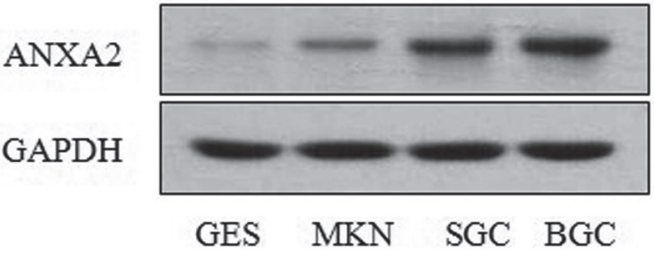

B

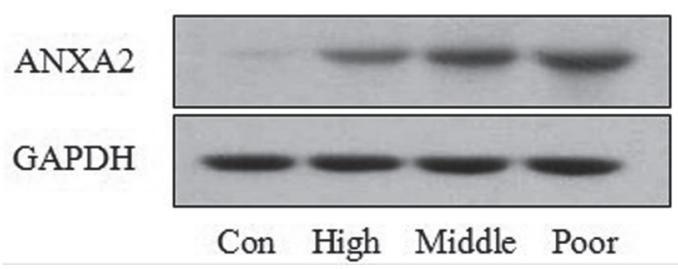

D
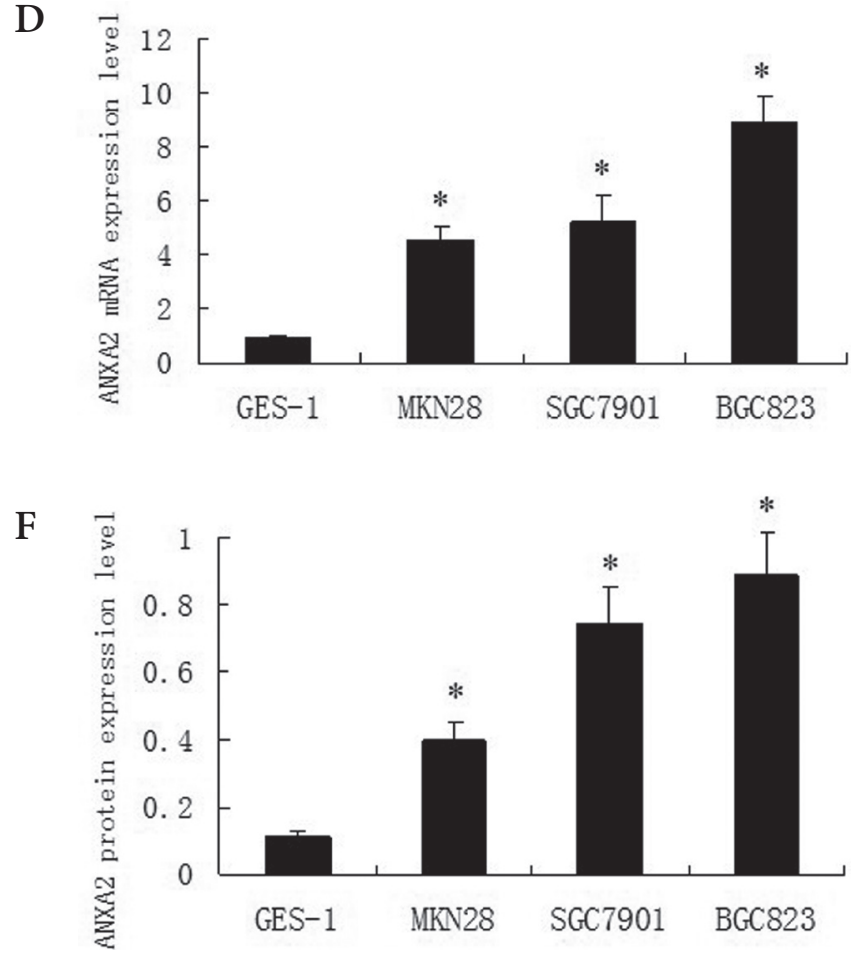

Figure 2. Expression of ANXA2 is associated with differentiation levels of GC tissue or cells.

Clinical gastric tumor specimens with differentiation stage of well, middle or poor differentiation as well as cancer-adjacent normal tissues were taken from GC patients and subjected to RT-QPCR (A) and western blotting (B and C) to determine the expression levels of ANXA2 mRNA or protein respectively in vivo. Normal control human gastric mucosa epithelial cell line GES-1 as well as three gastric cancer cell lines MKN28 (well differentiation), SGC7901 (middle differentiation) and BGC823 (poor differentiation) were subjected to RT-QPCR (D) and Western blotting (E and F) to determine the expression of ANXA2 in vitro. For RT-QPCR and Western blotting assays, GAPDH was used as an endogenous reference to standardize the mRNA and protein expression levels. Values are mean \pm S.D. $(n=3) .{ }^{*}, \mathbf{p}<0.05$ compared with cancer-adjacent normal tissues or GES-1 cells. The images shown are representative images from at least three independent experiments. 
$\mathrm{B}$ and $\mathrm{C}$, indicating that the expression level of ANXA2. was negative when correlated with the differentiation stage of gastric tumor.

To further verify the correlation of ANXA2 expression with GC differentiation and the feasibility of following study for mechanism, ANXA2 expression in GC cell lines (MKN28, SGC7901 and BGC823) with various differentiation stages were also detected by RT-QPCR and Western blotting analysis. The results were consistent with ANXA2 expression in differentiation stage of GC (Fig 2 D, E and F). Taken together, ANXA2 expression was negative when correlated with the differentiation level of gastric tumor in vivo and in vitro, suggesting the important function of ANXA2 in the pathological process of GC.

Expression of ANXA2 is upregulated in MDR gastric cancer cells. Multidrug resistance (MDR) to chemotherapeutic drugs is most common reason for clinical chemotherapy failure. Moreover, it is reported that drug sensitivity is related to the degrees of differentiation in cancer cells. To address the role of ANXA2 in the multidrug resistance of GC, the IC50 values of DDP in the SGC7901/DDP and parent SGC7901 cells were determined to establish the multidrug resistance cell line firstly. The results of SRB showed that the IC50 values of DDP is $(5.36 \pm 0.73) \mu \mathrm{g} / \mathrm{ml}$ and $(0.26$ $\pm 0.03) \mu \mathrm{g} / \mathrm{ml}$ in the SGC7901/DDP and SGC7901 cells, respectively, which indicated that the SGC7901/DDP cells were 23.4 times more resistant to DDP than SGC7901 cells (Fig. 3A). Further, we compared the expression of ANXA2 in DDP-resistant SGC7901/DDP cells and parent SGC7901 cells. RT-QPCR and western blotting analysis results revealed that the expression of ANXA2 was higher in SGC7901/DDP cells than that in its parent SGC7901 cells for both mRNA and protein levels (Fig. 3B, C and D). These data suggested that ANXA2 is associated with the development of MDR in gastric cancer cells.

ANXA2 siRNA reversed MDR of drug resistant cells. To further investigate the effects of ANXA2 in the multidrug resistance of GC, were employed. We first investigated the ability of ANXA2 to inhibit the expression of ANXA2 in SGC7901/DDP cells. Cells were transfected with different concentration of for different periods, and the results of RTQPCR and western blotting showed that ANXA2 knockdown the endogenous ANXA2 mRNA and protein expression effectively in concentration- (Fig. 4A, B and C) and time- (Fig. $4 \mathrm{D}, \mathrm{E}$ and $\mathrm{F}$ ) dependent manners. The optimal concentration is $80 \mu \mathrm{M}$, and the optimal period is $48 \mathrm{~h}$, suggesting that ANXA2 efficiently inhibited the transcription and translation of ANXA2 in SGC7901/DDP cells. Although SGC7901/DDP cells were selected with the chemotherapeutic drug cisplatin, they also displayed multiple resistances to other chemotherapeutic drugs. Therefore, we futher investigate the effects of ANXA2 on the drug sensitivity of SGC7901/DDP to one Pgp-related drug (doxorubicin) and on P-gp-non-related drugs (5-FU and cisplatin). As showed in Table 3, the IC50 values for doxorubicin, 5-fluorouracil, and cisplatin were all higher
A

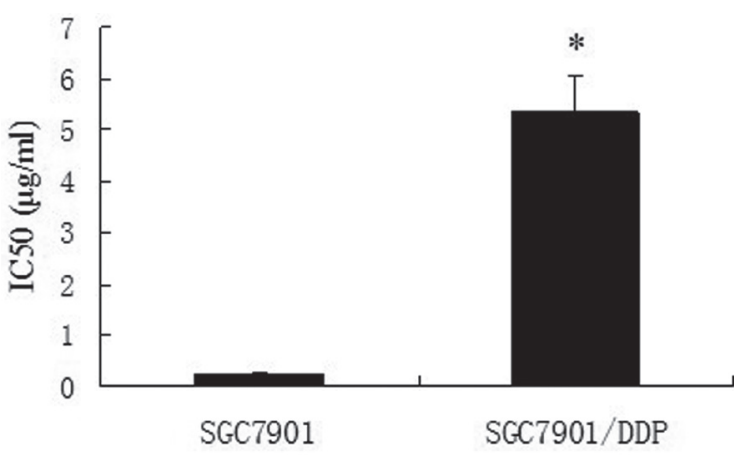

B

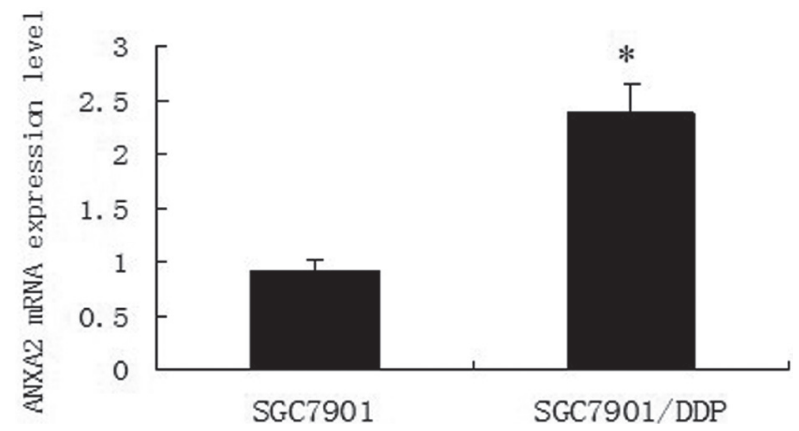

C

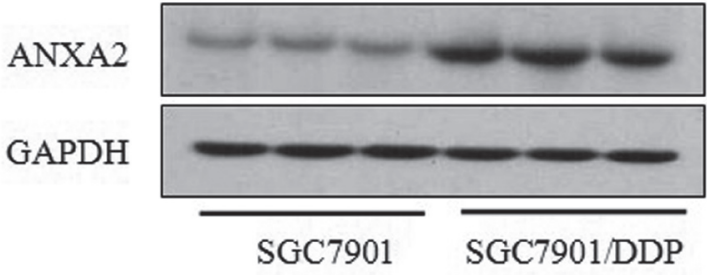

$\mathrm{D}$

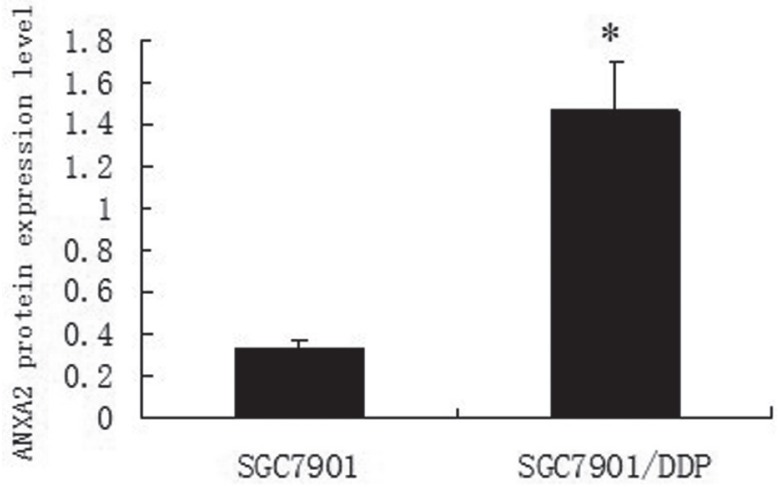

Figure 3. Expression of ANXA2 in MDR gastric cancer cells. After treatment with different concentrations of DDP, drug sensitivity of SGC7901/DDP and parent SGC7901 cells were assessed by SRB assay (A). The expression of ANXA2 was detected by RT-QPCR (B) and Western blotting (C and D). For RT-QPCR and Western blotting assays, GAPDH was used as an endogenous reference to standardize the mRNA and protein expression levels. Values are mean \pm S.D. $(n=3) .^{*}, p<0.05$ compared with SGC7901 cells group. The images showed representative images from at least three independent experiments. 
in SGC7901/DDP cells than that in its parent SGC7901 cells. The results showed that drug resistant drug resistant SGC7901 cells displayed cross resistance to these chemotherapeutic drugs. In addition, after transfection of $80 \mathrm{nM}$ ANXA2, the IC50 values for doxorubicin, 5-fluorouracil and cisplatin was decreased significantly compared with non-targeted siRNA and Lipofectamine 2000 control group ( $\mathrm{p}<0.05$ ), indicating that ANXA2 siRNA could partially reverse resistance to DDP in multi-drug resistant SGC7901 cells.

ANXA2 siRNA influenced the expression of MDR-related genes. To further explore the potential mechanism of ANXA2 on development of MDR, the MDR-related genes including P-gp, MRP1, Bcl-2, and Bax were detected by RT-QPCR and western blotting. As were shown in Figure 5,

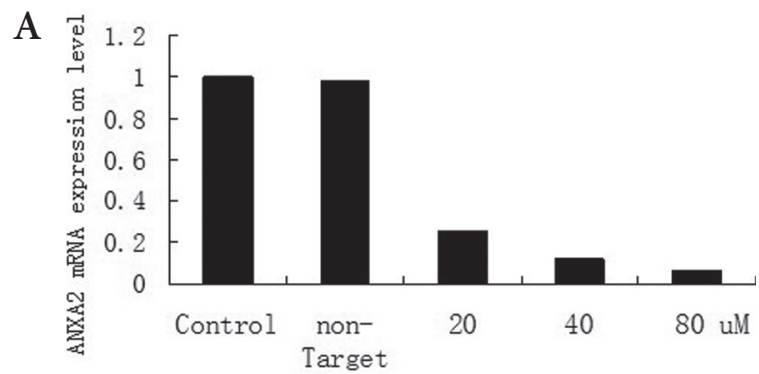

B

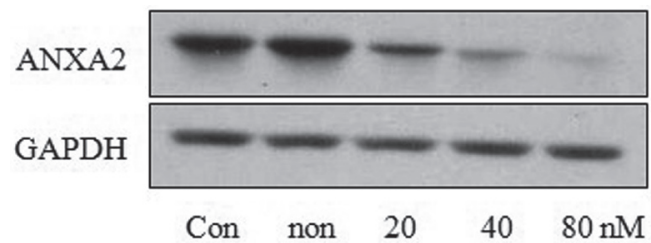

\section{C}

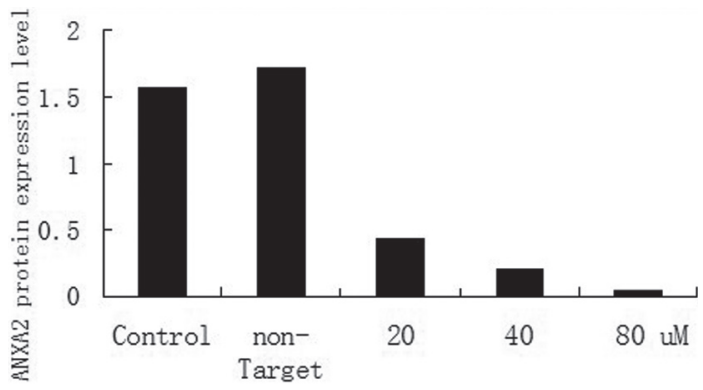

$\mathrm{E}$

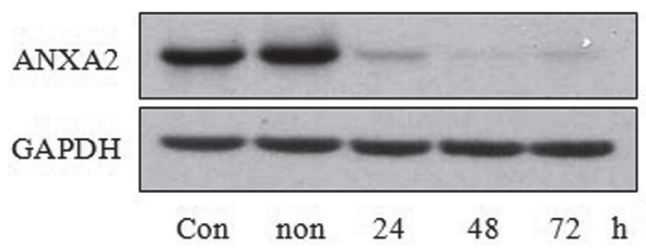

G

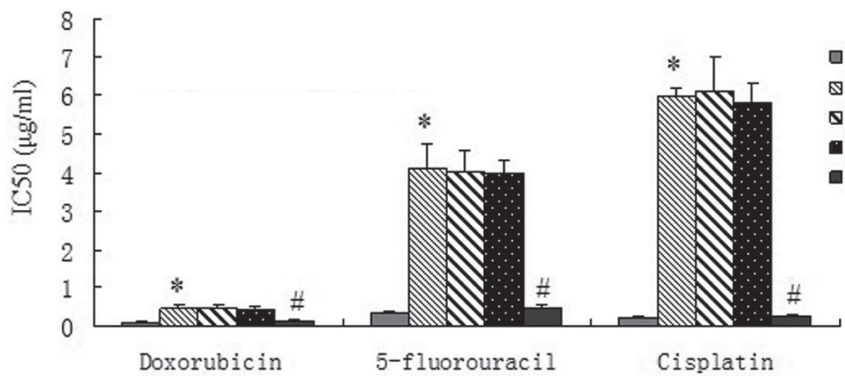

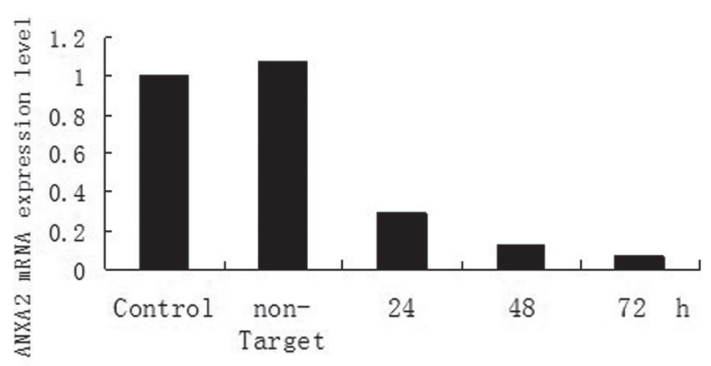

F

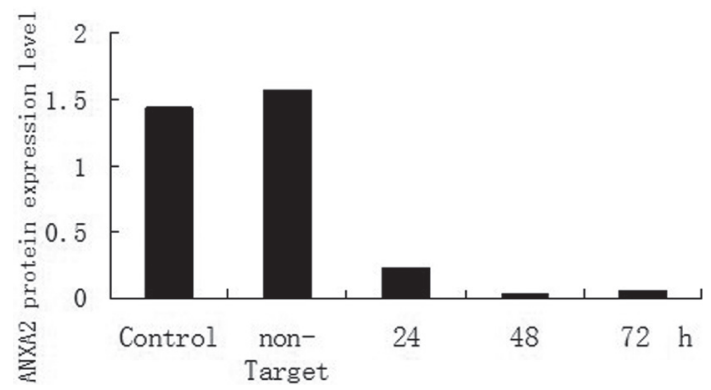

$\square$ SGC7901

\& SGC7901/DDP

$\Delta \mathrm{SGC7} 701 / \mathrm{DDP}+\mathrm{Lipo}$

- SGC7901/DDP+non-targeted-siRNA

- SGC7901/DDP+ANXA2-siRNA

Figure 4. Effects of ANXA2 siRNA on MDR of drug resistant cells SGC7901/DDP.

SGC7901/DDP cells were transfected with 20, 40 or $80 \mathrm{nM}$ of ANXA2- or non-targeted siRNA for $48 \mathrm{~h}$ (A, B and C), alternatively, $80 \mathrm{nM}$ siRNA for 24 , 48 or $72 \mathrm{~h}$ (D, E and F), and then were collected for RT-QPCR and Western blot assays to confirm the ANXA2 knockdown efficiency of ANXA2-siRNA. SGC7901/DDP cells were transfected with or without 80nM ANXA2-siRNA or non-targeted siRNA or treated only with Lipofectamine 2000 control for $48 \mathrm{~h}$, then SRB assays were performed to determine the IC50 values for chemotherapeutic drugs (Doxorubicin, 5-fluorouracil or Cisplatin) in SGC7901/ DDP cells and its control SGC7901 cells $(G)$. Values are mean \pm S.D. $(n=6) .{ }^{\star}, p<0.05$ compared with non-targeted siRNA group. 
Table 3. IC50 values for chemotherapeutic drugs in SGC7901/DDP cells. (means \pm SD, $n=6$ ).

\begin{tabular}{lccc}
\hline & Doxorubicin $(\boldsymbol{\mu g} / \mathrm{ml})$ & 5-fluorouracil $(\boldsymbol{\mu g} / \mathbf{m l})$ & Cisplatin $(\boldsymbol{\mu g} / \mathbf{m l})$ \\
\hline SGC7901 & $0.10+0.02$ & $0.32 \pm 0.06$ & $0.22 \pm 0.02$ \\
SGC7901/DDP & $0.46 \pm 0.07^{*}$ & $4.12 \pm 0.63^{*}$ & $5.96 \pm 0.21^{\star}$ \\
SGC7901/DDP+Lipo & $0.48 \pm 0.07$ & $4.01 \pm 0.58$ & $6.08 \pm 0.91$ \\
SGC7901/DDP+non-targeted-siRNA & $0.43 \pm 0.06$ & $3.98 \pm 0.33$ & $5.81 \pm 0.50$ \\
SGC7901/DDP+ANXA2-siRNA & $0.13 \pm 0.02^{*}$ & $0.47 \pm 0.08^{*}$ & $0.26 \pm 0.02^{*}$ \\
\hline
\end{tabular}

${ }^{*} P<0.05$ for SGC7901/DDP cells $v s$ SGC7901 cells; ${ }^{*} P<0.05$ for SGC7901/DDP+ANXA2-siRNA cells $v s$ SGC7901/DDP+non-targeted-siRNA cells and SGC7901/DDP+Lipo cells. Experiment was conducted in triplicate.

that the expression of P-gp, MRP1, Bcl-2 were significantly downregulated, while the expression of Bax was markedly upregulated by 80nM ANXA2 in SGC7901/DDP cells. However, several other MDR factors such as GST- $\pi$, TOPO-I and TOPO-II have no obvious changes. These suggested that the MDR reversal effect of ANXA2 in SGC7901/DDP cells may be caused by regulating the expression of P-gp, MRP1, Bcl-2 and Bax.

ANXA2 siRNA affected the phosphorylation of AKT and ERK in SGC7901/DDP cells. To determine whether the expression of ANXA2 was also involved in activation of some molecular in signaling pathway in SGC7901/DDP cells, Western blotting analysis of MAP kinases and AKT were performed. As were shown in Figure 6, the results revealed that phosphorylation of p38-MAPK and AKT, but not ERK1/2 or JNKs were specifically decreased in SGC7901/DDP after transfection of $80 \mathrm{nM}$ ANXA2, suggesting that the MDR reversal effect of ANXA2 in SGC7901/DDP cells may be related with inhibiting the activation of p-p38-MAPK and p-AKT.
A

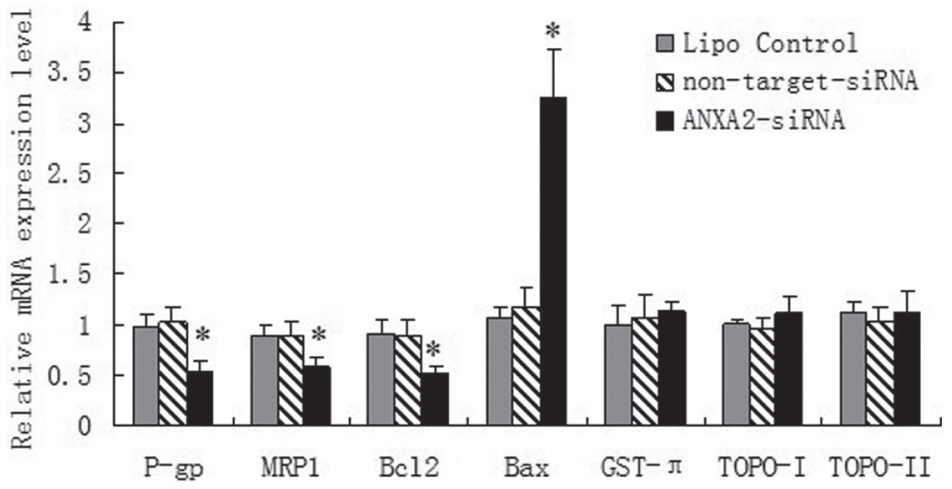

$\mathrm{C}$

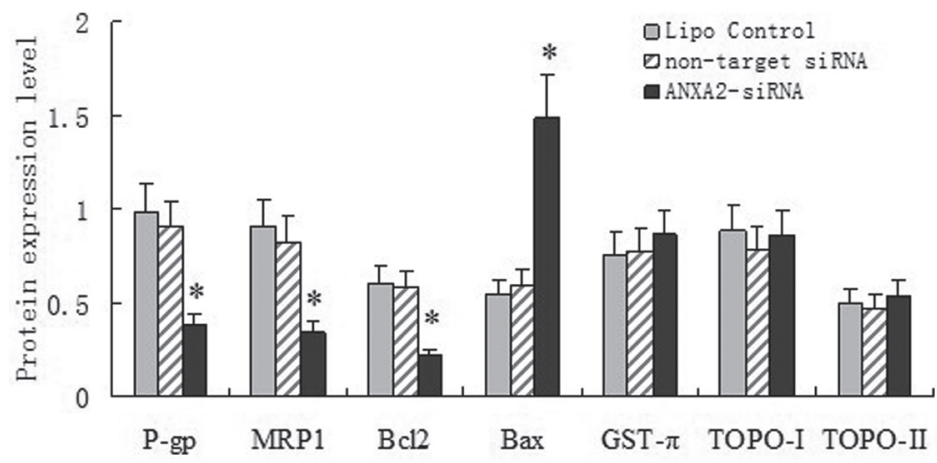

B

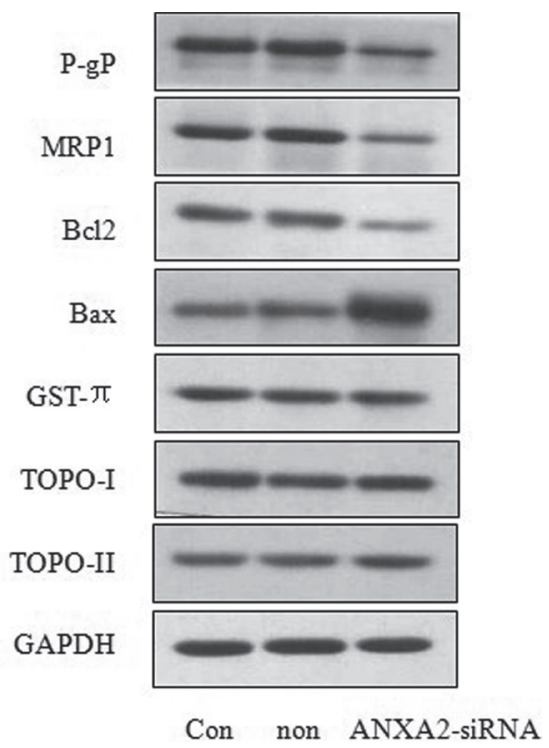

Figure 5. Effect of ANXA2 knockdown on the expression of MDR-related genes.

SGC7901/DDP cells were transfected with $80 \mathrm{nM}$ of ANXA2- or non-targeted or only treated with Lipofectamine 2000 for $48 \mathrm{~h}$, then were subjected to (A) RT-QPCR and Western blotting $(B, C)$ to detect mRNA and protein expression levels of MDR-related genes. GAPDH was used for an endogenous reference to standardize the $\mathrm{mRNA}$ and protein expression levels. Values are mean \pm S.D. $(\mathrm{n}=3)$. ${ }^{*}, \mathrm{p}<0.05$ compared with non-targeted group. The images shown are representative images from at least three independent experiments. 
A

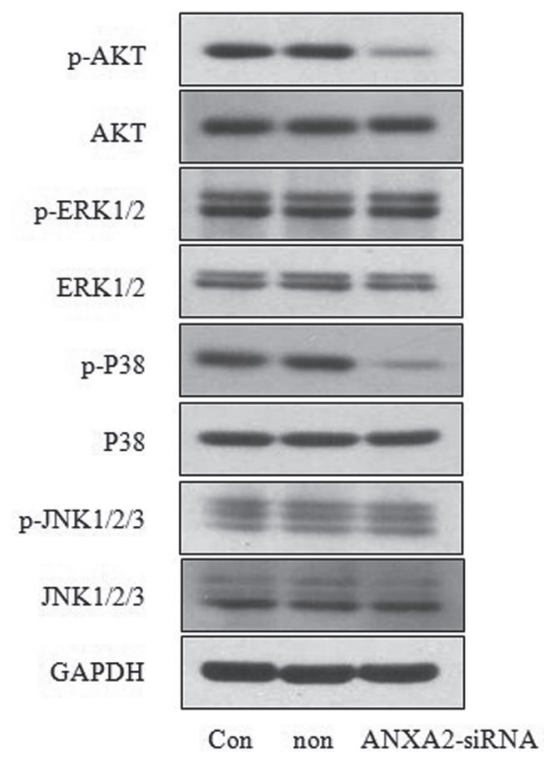

B

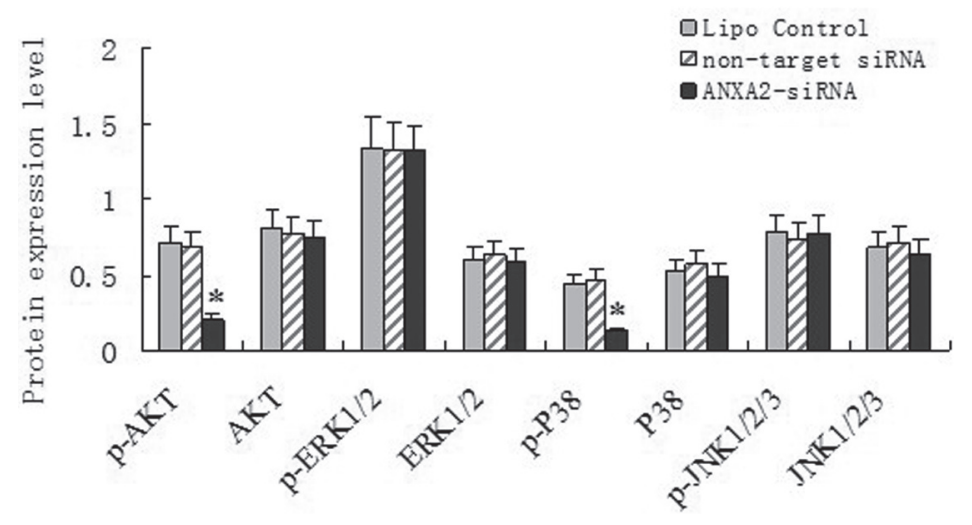

Figure 6. Effect of ANXA2 on the phosphorylation of of signaling pathway.

SGC7901/DDP cells were transfected with $80 \mathrm{nM}$ of ANXA2- or non-targeted siRNA or treated only with Lipofectamine 2000 for $48 \mathrm{~h}$, then were subjected to Western blotting $(A, B)$ to detect the protein expression levels of AKT, ERK, p38MAPK and JNKs as well as their phosphorylation levels. GAPDH was used as an endogenous reference to standardize the protein expression levels. Values are mean \pm S.D. $(n=3) .{ }^{*}, p<0.05$ compared with non-targeted siRNAs group. The images shown are representative images from at least three independent experiments.

P38MAPK and AKT inhibitor increased the drug sensitivity of SGC7901/DDP cells. In order to show whether P38MAPK or AKT phosphorylation functionally contribute to the drug resistance of SGC701/DDP cell line, drug sensitivity experiments using P38MAPK inhibitor SB203580 or AKT inhibitor triciribine were performed. The results showed that the two inhibitors could decrease the IC50 and increase the drug sensitivity of SGC701/DDP cells to doxorubicin, 5-fluor- ouracil and cisplatin in a similar way as ANXA2 siRNAs does, as were shown in Fig.7

\section{Discussion}

Several investigators have reported that up-regulation of ANXA2 is a characteristic feature of human hepatocellular carcinoma, colorectal cancer, breast cancer and ovarian
A

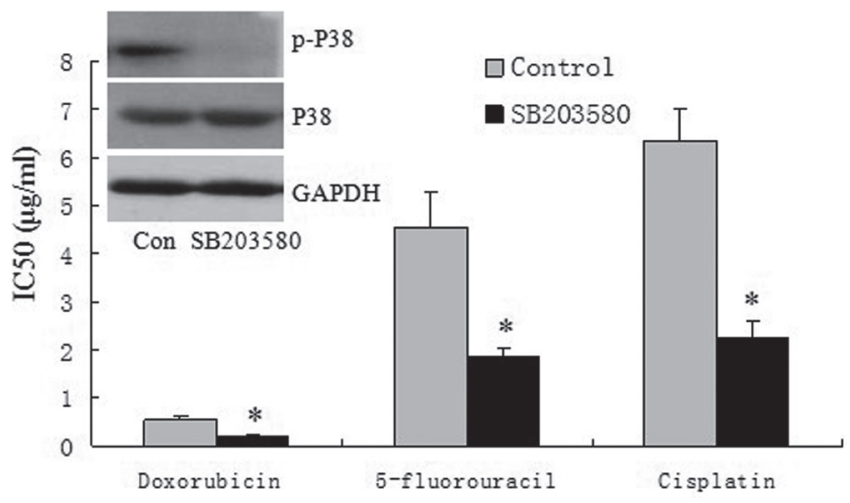

B

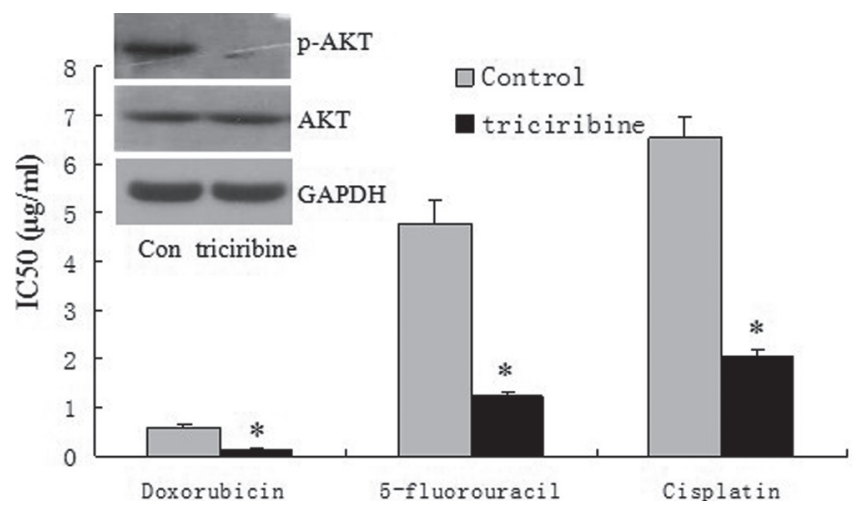

Figure 7. Effects of p38MAPK or AKT inhibitor on drug sensitivities in SGC7901/DDP cells SGC7901/DDP cells were treated with p38MAPK inhibitor SB203580 (A) or AKT inhibitor triciribine (B), then protein expression and phosphorylation of p38MAPK and AKT were detected by western bloting, and IC50 were determined by SRB assays. GAPDH was used for an endogenous reference to standardize the protein expression levels. Values are mean \pm S.D. $(n=3) .{ }^{*}, p<0.05$ compared with non-targeted siRNA group. The images shown are representative images from at least three independent experiments. 
cancer[16-19] . Conversely, others have shown that downregulation of ANXA2 has been reported in prostate carcinoma, esophageal carcinoma, and laryngeal squamous cell carcinoma [22-24]. Therefore, the role and mechanism of ANXA2 may be different in different types of tumors. Previous studies have reported that the expression of ANXA2 gene was significantly up-regulated in human GC. Because the expression of ANXA2 in GC was significantly associated with depth of invasion, lymphnode metastasis and distant metastasis, TNM stage, it could be considered as a useful prognostic marker to predict tumor progression and prognosis in gastric cancer $[25,26]$. In the present study, ANXA2 was found to be up-regulated in gastric cancer tissues.

The development of MDR is a major obstacle to the effective chemotherapy of gastric cancer. In addition, the MDR characteristic is related with the degree of cancer differentiation. Usually, poorly differentiated carcinoma is more resistant to the chemotherapeutic agents [27]. Therefore, new treatment strategies are in urgent. We found that ANXA2 was negative correlated with the differentiation degree of gastric cancer both in clinical specimens of gastric carcinoma and GC cell lines (MKN28, SGC7901 and BGC823) with various differentiation stage.

Previous data suggest that ANXA2 may take part in mechanism of MDR in bladder and breast cancer [28, 29]. To explore the effect and mechanism of ANXA2 on MDR in GC, we successfully established a DDP resistant cell line SGC7901/DDP after continual exposure of SGC7901 to cisplatin, a major chemotherapy drug in gastric carcinoma treatment [30].The results showed that the resistance index was 23.4. The establishment of SGC7901/DDP cells has provided a powerful tool for further study of the effect and mechanism of ANXA2 on MDR. The recent report showed that the expression of ANXA2 was increaed in resistant MCF7/ADR cells, but not in MCF-7 cells [28]. In the present study, we found that the expression of ANXA2 was significantly higher in SGC7901/ DDP cells than in SGC7901 cells, indicating that ANXA2 played a key role in the development of MDR of gastric cancer.

Due to the advantages of high specificity and efficiency, small interfering RNA (siRNA) technique has become a popular reverse genetic method for gene function research [31]. To further investigate the effect of ANXA2 on the development of MDR of GC, ANXA2 siRNA was employed to inhibit the endogenous expression of ANXA2. The results showed that inhibition of endogenous ANXA2 in SGC7901/DDP cells enhanced sensitivity to doxorubicin (P-gp-related drug) and cisplatin, 5-fluorouracil (P-gp-nonrelated drug). These findings suggest that ANXA2 siRNA reversed MDR of human GC cells.

High expression of MDR proteins in cancer cells is a major reason of recurrence after treatment. Previous stud has described a relationship between the expression of adenosine 5 '-triphosphate (ATP)-binding cassette (ABC) proteins and the poor prognosis of cancer patients treated with chemotherapy
[32]. MDR proteins such as P-glycoprotein (P-gp)/ABCB1 and Multiple Drug Resistant Protein 1 (MRP1) are recognized for its role in removing a variety of chemotherapeutic agents from the cancer cells, thereby preventing cell death [33]. In GC, drug resistance has also been associated with altered expression of anti-apoptotic members of the Bcl-2 [34]. ANXA2 siRNA increased the expression of Bax, as well as decreased the expression of P-gp, MRP1, and Bcl-2. These data suggested that ANXA2 siRNA allowed more chemotherapeutic agents into cells and induced apoptosis.

Several signaling pathways including: PI3K/Akt, ERK1/2, p38-MAPK and JNK signaling pathway were involved in the development of MDR [35-38]. Abnormal activation of these pathways might be responsible for the modulation of P-glycoprotein-mediated and P-glycoprotein-unmediated multidrug resistance in many different human cancer cells. Therefore, all of them were concerned as promising targets for therapeutic intervention. For example, Liang J et al reported that inhibition of PI3K/Akt led to the inhibition of $\operatorname{PrP}(\mathrm{C})$-induced drug resistance in GC cell [35]. In our study, we found that activation of $\mathrm{p} 38$-MAPK and AKT signaling pathway was inhibited by the ANXA2 siRNA transfection in resistant GC cells. Our results suggested that drug resistance in SGC7901/DDP cells is attributed at least partially to the activation of p38-MAPK and AKT signal pathway and inhibition of these pathways could increase the sensitivity of SGC7901/DDP to P-gp-unrelated drugs as well as P-gp-related drug.

\section{Conclusion}

In summary, in this report, we show that ANXA2 is upregulated in gastric cancer and resistance of GC cell to DDP could be reversed by silencing ANXA2. Its possible mechanism may be related with the changes in the expressions of P-gp, MRP1, Bcl-2, and Bax, as well as activation of the p38-MAPK and AKT. These results may provide new strategies for reversal of multidrug resistance in human gastric cancer cells.

Acknowledgments: This work was supported by grants from the National Natural Science Foundations of China ((No.81072033 to Yong Li; No. 81372580 to Yong Li) and Hebei Natural Science Foundation of China (No. C2010000619 to Yong Li); Extra Characteristic Foundation of Colleges and Universities in Hebei Province (NO. [2005]52, to Yong Li)

\section{References}

[1] JEMAL A, BRAY F, CENTER MM, FERLAY J, WARD E et al: Global cancer statistics. CA Cancer J Clin 2011; 61: 69-90. http://dx.doi.org/10.3322/caac.20107

[2] MCCRACKEN M, OLSEN M, CHEN MS JR, JEMAL A, THUN $\mathrm{M}$ et al: Cancer incidence, mortality, and associated risk factors among Asian Americans of Chinese, Filipino, Vietnamese, Korean, and Japanese ethnicities. CA Cancer J Clin 2007; 57: 190-205. http://dx.doi.org/10.3322/canjclin.57.4.190 
[3] ZHAO P, DAI M, CHEN W, LI N: Cancer trends in China. Jpn J Clin Oncol 2010; 40: 281-285. http://dx.doi.org/10.1093/ jico/hyp187

[4] QIU MZ, WANG ZQ, LUO HY, ZHANG DS, ZHOU ZW et al: Prognostic analysis in node-negative gastric cancer patients in China. Tumour Biol 2011; 32: 489-492. http://dx.doi. org/10.1007/s13277-010-0142-5

[5] NISHIYAMA M: Chemotherapy for gastric cancer in Japan. Int J Clin Oncol 2008; 13: 191-192. http://dx.doi.org/10.1007/ s10147-008-0790-2

[6] CUNNINGHAM D, ALluM WH, STENNing SP, THOMPSON JN, VAN DE VELDE CJ et al: Perioperative chemotherapy versus surgery alone for resectable gastroesophageal cancer. N Engl J Med 2006; 355: 11-20. http://dx.doi.org/10.1056/NEJMoa055531

[7] CERVANTES A, RODA D, TARAZONA N, ROSELLO S, PEREZ-FIDALGO JA: Current questions for the treatment of advanced gastric cancer. Cancer Treat Rev 2013; 39: 60-67. http://dx.doi.org/10.1016/j.ctrv.2012.09.007

[8] BROXTERMAN HJ, GOTINK KJ, VERHEUL HM: Understanding the causes of multidrug resistance in cancer: a comparison of doxorubicin and sunitinib. Drug Resist Updat 2009; 12: 114-126. http://dx.doi.org/10.1016/ j.drup.2009.07.001

[9] MAI J, WAISMAN DM, SLOANE BF: Cell surface complex of cathepsin B/annexin II tetramer in malignant progression. Biochim Biophys Acta 2000; 1477: 215-230. http://dx.doi. org/10.1016/S0167-4838(99)00274-5

[10] OLIFERENKO S, PAIHA K, HARDER T, GERKE V, SCHWARZLER C et al: Analysis of CD44-containing lipid rafts: Recruitment of annexin II and stabilization by the actin cytoskeleton. J Cell Biol 1999; 146: 843-854. http://dx.doi. org/10.1083/jcb.146.4.843

[11] SURETTE AP, MADUREIRA PA, PHIPPS KD, MILLER VA, SVENNINGSSON P et al: Regulation of fibrinolysis by S100A10 in vivo. Blood 2011; 118: 3172-3181. http://dx.doi. org/10.1182/blood-2011-05-353482

[12] ZHAO P, ZHANG W, WANG SJ, YU XL, TANG J et al: HAb18G/CD147 promotes cell motility by regulating annexin II-activated RhoA and Racl signaling pathways in hepatocellular carcinoma cells. Hepatology 2011; 54: 2012-2024. http://dx.doi.org/10.1002/hep.24592

[13] WOODHAM AW, DA SILVA DM, SKEATE JG, RAFF AB, AMBROSO MR et al: The S100A10 subunit of the ANXA2 heterotetramer facilitates L2-mediated human papillomavirus infection. PLoS One 2012; 7: e43519. http://dx.doi. org/10.1371/journal.pone.0043519

[14] MARTIN M, LEFFLER J, BLOM AM: ANXA2 and A5 serve as new ligands for C1q on apoptotic cells. J Biol Chem 2012; 287: 33733-33744. http://dx.doi.org/10.1074/jbc.M112.341339

[15] MADUREIRA PA, HILL R, MILLER VA, GIACOMANTONIO C, LEE PW et al: ANXA2 is a novel cellular redox regulatory protein involved in tumorigenesis 2011, 2: 1075-1093.

[16] SUN Y, GAO G, CAI J, WANG Y, QU X et al: ANXA2 is a discriminative serological candidate in early hepatocellular carcinoma. Carcinogenesis 2013; 34: 595-604. http://dx.doi. org/10.1093/carcin/bgs372
[17] YANG T, PENG H, WANG J, YANG J, NICE EC et al: Prognostic and diagnostic significance of ANXA2 in colorectal cancer. Colorectal Dis 2013; 15: e373-381. http://dx.doi. org/10.1111/codi.12207

[18] DENG S, JING B, XING T, HOU L, YANG Z: Overexpression of ANXA2 is associated with abnormal ubiquitination in breast cancer. Genomics Proteomics Bioinformatics 2012; 10: 153-157. http://dx.doi.org/10.1016/j.gpb.2011.12.001

[19] LOKMAN NA, ELDER AS, WEEN MP, PYRAGIUS CE, HOFFMANN P et al: ANXA2 is regulated by ovarian cancer-peritoneal cell interactions and promotes metastasis. Oncotarget 2013; 4: 1199-1211.

[20] ZHANG Q, YE Z, YANG Q, HEX, WANG H et al: Upregulated expression of annexin II is a prognostic marker for patients with gastric cancer. World J Surg Oncol 2012; 10: 103. http:// dx.doi.org/10.1186/1477-7819-10-103

[21] LI W, LI L, LIU Z, LIU C, LIU Z et al: Expression of the full-length telomerase reverse transcriptase (hTERT) transcript in both malignant and normal gastric tissues. Cancer Lett 2008; 260: 28-36. http://dx.doi.org/10.1016/ j.canlet.2007.10.018

[22] LIU JW, SHEN JJ, TANZILLO-SWARTS A, BHATIA B, MALDONADO CM et al: Annexin II expression is reduced or lost in prostate cancer cells and its re-expression inhibits prostate cancer cell migration. Oncogene 2003; 22: 1475-1485. http://dx.doi.org/10.1038/sj.onc.1206196

[23] QI YJ, HE QY, MA YF, DU YW, LIU GC et al: Proteomic identification of malignant transformation-related proteins in esophageal squamous cell carcinoma. J Cell Biochem 2008; 104: 1625-1635. http://dx.doi.org/10.1002/jcb.21727

[24] PENA-ALONSO E, RODRIGO JP, PARRA IC, PEDRERO JM, MEANA MV et al: ANXA2 localizes to the basal epithelial layer and is down-regulated in dysplasia and head and neck squamous cell carcinoma. Cancer Lett 2008; 263: 89-98. http://dx.doi.org/10.1016/j.canlet.2007.12.029

[25] ZHANG Q, YE Z, YANG Q, HEX, WANG H et al: Upregulated expression of annexin II is a prognostic marker for patients with gastric cancer. World J Surg Oncol 2012; 10: 103. http:// dx.doi.org/10.1186/1477-7819-10-103

[26] EMOTO K, SAWADA H, YAMADA Y, FUJIMOTO H, TAKAHAMA Y et al: Annexin II overexpression is correlated with poor prognosis in human gastric carcinoma. Anticancer Res 2001; 21: 1339-1345.

[27] LI Y, TAN BB, ZHAO Q, FAN LQ, LIU Y et al: Tumor chemosensitivity is correlated with expression of multidrug resistance associated factors in variously differentiated gastric carcinoma tissues. Hepatogastroenterology 2013; 60: 213-216.

[28] ZHANG F, ZHANG L, ZHANG B, WEI X, YANG Y et al: Anxa2 plays a critical role in enhanced invasiveness of the multidrug resistant human breast cancer cells. J Proteome Res 2009; 8: 5041-5047. http://dx.doi.org/10.1021/pr900461c

[29] MENG Q, LEI T, ZHANG M, ZHAO J, ZHAO XH et al: Identification of proteins differentially expressed in adriamycin-resistant (pumc-91/ADM) and parental (pumc-91) human bladder cancer cell lines by proteome analysis. J Cancer Res Clin Oncol 2013; 139: 509-519. http://dx.doi.org/10.1007/ s00432-012-1350-8 
[30] PIACENTINI P, DURANTE E, TROLESE A, MERCANTI A, BONETTI A: Weekly Taxotere and cisplatin with continuousinfusion 5-fluoruracil for the treatment of advanced gastric and esophageal cancer: a prospective, observational, singleinstitution experience. Gastric Cancer 2012; 15: 106-110. http://dx.doi.org/10.1007/s10120-011-0078-5

[31] HANNON GJ: RNA interference. Nature 2002; 418: 244-251. http://dx.doi.org/10.1038/418244a

[32] LUCIO KA, ROCHA GDA G, MONCAO-RIBEIRO LC, FERNANDES J, TAKIYA CM et al: Oleanolic acid initiates apoptosis in non-small cell lung cancer cell lines and reduces metastasis of a B16F10 melanoma model in vivo. PLoS One 2011; 6: e28596. http://dx.doi.org/10.1371/journal. pone.0028596

[33] SHAROM FJ: ABC MULTIDRUG TRANSPORTERS: structure, function and role in chemoresistance. Pharmacogenomics 2008; 9: 105-127. http://dx.doi.org/ 10.2217/14622416.9.1.105

[34] WANG Y, LIU L, LIU X, ZHANG H, LIU J et al: Shugoshin1 enhances multidrug resistance of gastric cancer cells by regulating MRP1, Bcl-2, and Bax genes. Tumour Biol 2013;
34: 2205-2214. http://dx.doi.org/10.1007/s13277-0130758-3

[35] LIANG J, GE F, GUO C, LUO G, WANG X et al: Inhibition of PI3K/Akt partially leads to the inhibition of $\operatorname{PrP}(\mathrm{C})$ induced drug resistance in gastric cancer cells. FEBS J 2009; 276: 685-694. http://dx.doi.org/10.1111/j.17424658.2008.06816.x

[36] TAKEUCHI A, ETO M, SHIOTA M, TATSUGAMI K, YOKOMIZO A et al: Sunitinib enhances antitumor effects against chemotherapy-resistant bladder cancer through suppression of ERK1/2 phosphorylation. Int J Oncol 2012; 40: 1691-1696.

[37] GUO X, MA N, WANG J, SONG J, BU X et al: Increased p38MAPK is responsible for chemotherapy resistance in human gastric cancer cells. BMC Cancer 2008; 8: 375. http://dx.doi. org/10.1186/1471-2407-8-375

[38] ZHU MM, TONG JL, XU Q, NIE F, XU XT et al: Increased JNK1 signaling pathway is responsible for ABCG2-mediated multidrug resistance in human colon cancer. PLoS One. 2012, 7(8): e41763. http://dx.doi.org/10.1371/journal. pone. 0041763 